\title{
流体动力学作用对粒子聚集影响的布朗动力学模拟
}

\author{
李旭徐升华孙祉伟* \\ (中国科学院微重力重点实验室, 中国科学院力学研究所, 北京 100190)
}

\begin{abstract}
摘要: 采用布朗动力学模拟方法, 研究了流体动力学作用对稀溶液中悬浮粒子聚集过程的影响. 模拟中忽略了 一个粒子同时与多个粒子碰撞聚集的可能, 引人了前人有关两粒子间流体动力学作用影响的研究成果. 模拟结 果证实了流体动力学的作用在比较大的幅度上减缓了粒子的聚集过程, 是导致粒子聚集速率的实验值低于 Smoluchowski 理论值的重要原因之一. 另外, 在分别加人和排除重力作用, 以及考虑和忽略粒子间流体动力学作 用在内的各种条件下模拟了粒子的聚集过程, 得到了两种因素相互耦合作用时各因素对粒子聚集过程影响的结 果, 并从动力学的角度对这些因素的影响机制进行了相应的讨论.
\end{abstract}

关键词：布朗动力学方法; 流体动力学作用; 悬浮粒子; 稀溶液; 聚集 中图分类号：0641；0648

\section{Brownian Dynamics Simulation of the Influence of Hydrodynamic Interaction on Particle Coagulation}

\author{
LI Xu XU Sheng-Hua SUN Zhi-Wei* \\ (Key Laboratory of Microgravity (National Microgravity Laboratory), Institute of Mechanics, \\ Chinese Academy of Sciences, Beijing 100190, P. R. China)
}

\begin{abstract}
Brownian dynamics method was used to simulate the influence of hydrodynamic interaction on the suspension particle coagulation process in a dilute solution. In the simulation, the possibility of one particle colliding simultaneously with more than two particles was ignored and results from previous studies concerned with the hydrodynamic interaction between two particles were used. Our simulations confirm that the hydrodynamic interaction slows the coagulation process down greatly and this is an important reason for the experimental values of the coagulation rate being significantly less than those predicted by Smoluchowski theory. In addition, the particle coagulation process was simulated under varying conditions of combined two factors: gravity and hydrodynamic interactions. Results of how each factor affecting the particle coagulation process were obtained when the two factors were coupled. The mechanism responsible for these effects is discussed from a dynamics point of view.
\end{abstract}

Key Words: Brownian dynamic method; Hydrodynamic interaction; Particles in suspension; Diluted solution; Coagulation

胶体分散体系的聚集行为广泛地存在于人们的 生产实践和日常生活中, 因此对其研究有着重要的 意义 ${ }^{[1-25]}$. Smoluchowski ${ }^{[1,2]}$ 在其研究中给出了粒子在 极稀溶液中聚集初期的快聚集速率理论值. 然而, 迄
今为止很多的研究结果表明 ${ }^{[26,27]}$, 在实验中测得的粒 子聚集速率远低于 Smoluchowski 理论推导的结 果 ${ }^{[1,2]}$. 对此, 各国研究人员从不同角度进行了解释, 其中一些研究者怀疑这是由于重力的影响 ${ }^{[3-5]}$, 但是

Received: June 6, 2009; Revised: July 14, 2009; Published on Web: September 2, 2009.

*Corresponding author. Email: sunzw@imech.ac.cn; Tel: +86-10-82544093; Fax: +86-10-82544096.

The project was supported by the National Natural Science Foundation of China (10672173, 10432060, 20473108) and Knowledge Innovation Project of Chinese Academy of Sciences (0518181162).

国家自然科学基金(10672173, 10432060, 20473108)和中国科学院知识创新工程(0518181162)资助项目

C Editorial office of Acta Physico-Chimica Sinica 
更多的研究者认为是由于粒子之间存在流体动力 学作用(hydrodynamic interaction)的原因 ${ }^{[6-17]}$. 流体 动力学作用是指当流体中存在多个粒子时, 粒子 间扩散时通过流体而互相影响的作用. 为了对流 体动力学作用的影响有更加明确的认识, 本工作 中采用计算机模拟方法对其进行研究. 前人模拟 多粒子间的流体动力学作用时, 常采用扩散矩阵 计算粒子的位移,这种处理方法消耗了大量时间 计算距离较远而影响较小的粒子之间的作用, 计 算量过大. 在本工作中, 根据研究对象为稀释溶液 的特点, 将 Spielman ${ }^{[8]}$ 和 Honig 等 ${ }^{[9]}$ 处理两粒子间 流体动力学作用的研究成果应用到多粒子模拟中 去, 对流体动力学作用对粒子聚集的影响进行了 研究. 另一方面, 在真实环境中往往同时存在流体 动力学作用、重力和粒子间作用势等多个因素的 耦合影响, 这也是本工作中重要的研究内容. 此前 介绍的理论推导的方法 ${ }^{[1,2]}$ 有很大局限性, 只能对 粒子聚集的初始阶段短时间内单纯研究流体动力 学作用时的粒子聚集速率进行预测, 既不能研究 长期的粒子聚集过程, 也不能研究包含重力等多 种因素的耦合影响, 更无法描述聚集过程中粒子 的动力学行为. 而本工作中采用的模拟方法则可 以研究包括重力在内的多种因素耦合作用时长期 的粒子聚集过程, 也可以描述粒子在聚集过程中 的动力学行为. 通过进一步对比模拟结果, 认识了 流体动力学作用和重力因素在粒子聚集过程中的 影响.

\section{1 模拟方法与理论模型}

作为一种简洁有效的研究手段, 计算机模拟方 法广泛地应用在粒子聚集过程的研究中 ${ }^{[20-25,28-30]}$, 其 中较为常用的包括分子动力学(MD)方法和布朗动 力学(BD)方法等 ${ }^{[25,28-30]}$. 对分散体系各组分从分子层 次进行模拟无疑是最准确的模拟方法. 然而, 胶体分 散体系中存在液体分子和比其大几个数量级的胶体 粒子. 两者的运动特征量相差悬殊, 胶体粒子对应的 特征时间和特征长度较大, 水分子对应的特征时间 和特征长度较小. 本研究中关注的是胶体粒子的动 力学行为, 而不关心与胶体粒子无关的水分子之间 的运动和作用细节. 因此可以采用布朗动力学方法, 将流体分子对于胶体粒子运动的贡献做平均化处 理, 以布朗运动项表示. 这样在模拟程序中就不必照 顾流体分子去采用其对应的较小的时间步长, 而直
接采用胶体粒子对应的较大的时间步长来进行计 算, 从而大大降低了模拟中的计算量, 显著地提高了 计算效率.

在 BD 方法中, 选用的运动微分方程是朗之万

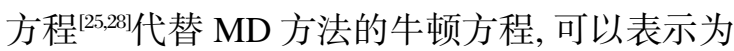

$$
\mathrm{d} p / \mathrm{d} t=F(t)-\gamma p(t)+R^{\prime}(t)
$$

在式(1)中, $p(t)$ 为粒子的动量, $F(t)$ 为粒子受到的保 守力, 而 $\gamma p(t)$ 和 $R^{\prime}(t)$ 两项分别代表耗散力和随机 力. 根据朗之万方程, 在计算机模拟程序中, 第 $i$ 个 粒子在 $t$ 时刻的位置 $r(t)$ 和经过时间步长 $\Delta t$ 后的位 置 $r(t+\Delta t)$ 有如下关系 ${ }^{[28]}$ :

$$
r_{i, n}(t+\Delta t)=r_{i, n}(t)+\frac{D_{0}}{k_{\mathrm{B}} T} F_{i, n}(t) \Delta t+\Delta r_{i, n}^{\mathrm{G}}
$$

这里 $n=1,2,3$, 为笛卡尔坐标系下相互正交的三个 方向, $D_{0}$ 为单粒子在溶液中的扩散系数, $k_{\mathrm{B}}$ 为 Boltzmann 常数, $T$ 为温度. 等式右边第二项代表由 于粒子受到外力而产生的位移, $\Delta r^{\mathrm{G}}$ 代表粒子在溶液 中作布朗运动的随机位移, 服从高斯分布: $\left\langle\left(\Delta r_{i, n}^{\mathrm{G}}\right)^{2}\right\rangle=$ $2 D_{0} \Delta t$, 其中 $D_{0}$ 服从 Stokes-Einstein 关系:

$$
D_{0}=k_{\mathrm{B}} T / 6 \pi \eta a
$$

式(2)中, $\eta$ 为溶液的粘度, $a$ 为粒子半径.

之前的研究者对分散体系中的流体动力学作用 进行模拟时, 是利用扩散矩阵, 无论粒子间距是多 少, 计算所有粒子两两之间的相互作用, 这就意味着 计算粒子位移时浪费了大量的时间在距离较远而影 响较小的粒子上. 我们的研究目标包括解释流体动

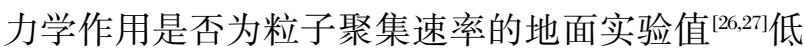
于 Smoluchowski 理论值 ${ }^{[1,2]}$ 的原因一一流体动力学 作用的存在是否会减缓分散体系中粒子的聚集,这 就意味着模拟的条件应该与 Smoluchowski 公式 $[1,2]$ 推导时采用的条件尽量保持一致, 即满足以下几个 条件: 溶液非常稀释, 粒子的浓度和数密度非常低, 粒子间平均距离很大, 多个粒子同时碰撞,一起聚集 的可能性很小, 也就是说只需要考虑两粒子碰撞聚 集的情形即可. Smoluchowski理论 ${ }^{[1,2]}$ 成立的条件还包 括: 在聚集初期, 溶液中的粒子均为单分散的, 即所 有粒子形状大小一致. 这意味着只需要处理一样大 小的粒子聚集即可. 综上所述, 需要解决的问题不再 是处理多个不同大小的粒子之间的聚集，而是简化 为处理两个同样大小的粒子之间的聚集. 此时可以 直接将 Spielman ${ }^{[8]}$ 和 Honig 等基于流体动力学的基 本原理处理两粒子扩散的研究结果应用在模拟中.

根据 Spielman 的研究成果 ${ }^{[8]}$, 可知存在流体动 
力学作用时, 两粒子之间的相对扩散系数 $D_{12}$ 可表 示为

$$
D_{12}=k_{\mathrm{B}} T / \zeta
$$

$\zeta$ 可以表示为

$$
\zeta=\frac{\kappa_{1} \kappa_{2}-\lambda_{1} \lambda_{2}}{\kappa_{1}+\kappa_{2}-\lambda_{1}-\lambda_{2}}
$$

$\kappa_{1}, \kappa_{2}, \lambda_{1}$ 和 $\lambda_{2}$ 的表达式为

$$
\begin{aligned}
\kappa_{1}= & \frac{4 \Gamma}{\Lambda}\left\{-2(2 n+3)(2 n-1) \sinh \left(\left(n+\frac{1}{2}\right)(\alpha-\beta)\right) \cdot\right. \\
& \exp \left(-\left(n+\frac{1}{2}\right)(\alpha+\beta)\right)+(2 n+1)[(2 n+3) \cdot \\
& \sinh \left(\left(n+\frac{3}{2}\right)(\alpha-\beta)\right) \exp \left(-\left(n-\frac{1}{2}\right)(\alpha+\beta)\right)+ \\
& (2 n-1) \sinh \left(\left(n-\frac{1}{2}\right)(\alpha-\beta)\right) \cdot \\
& \left.\left.\exp \left(-\left(n+\frac{3}{2}\right)(\alpha+\beta)\right)\right]\right\}
\end{aligned}
$$$$
\kappa_{2}=\frac{4 \Gamma}{\Lambda}\left\{-2(2 n+3)(2 n-1) \sinh \left(\left(n+\frac{1}{2}\right)(\alpha-\beta)\right) \cdot\right.
$$$$
\exp \left(\left(n+\frac{1}{2}\right)(\alpha+\beta)\right)+(2 n+1)[(2 n+3) \cdot
$$$$
\sinh \left(\left(n+\frac{3}{2}\right)(\alpha-\beta)\right) \exp \left(\left(n-\frac{1}{2}\right)(\alpha+\beta)\right)+
$$$$
(2 n-1) \sinh \left(\left(n-\frac{1}{2}\right)(\alpha-\beta)\right) \text {. }
$$$$
\left.\left.\exp \left(\left(n+\frac{3}{2}\right)(\alpha+\beta)\right)\right]\right\}
$$

$$
\lambda_{1}=\lambda_{2}=\frac{2 \Gamma}{\Lambda}\{8(1-\exp (-(2 n+1)(\alpha-\beta)))+(2 n+1) \cdot
$$$$
\sinh (\alpha-\beta) \cdot\{(2 n+3)[(2 n+1) \exp (\alpha-\beta)-(2 n-1) \cdot
$$$$
\exp (\alpha+\beta)]+(2 n-1)[(2 n+1) \exp (-(\alpha-\beta))-
$$$$
(2 n+3) \exp (-(\alpha+\beta))]\}\}
$$

式中的 $\Lambda$ 和 $\Gamma$ 为

$$
\begin{aligned}
& \Lambda=4 \sinh ^{2}\left[\left(n+\frac{1}{2}\right)(\alpha-\beta)\right]-(2 n+1)^{2} \sinh ^{2}(\alpha-\beta) \\
& \Gamma=\frac{a_{0}^{2} n(n+1)}{(2 n-1)(2 n+1)(2 n+3)}
\end{aligned}
$$

$\alpha$ 和 $\beta$ 分别满足:

$$
\begin{gathered}
\alpha=\ln \left[\frac{\left(r / a_{1}\right)^{2}-\left(a_{2} / a_{1}\right)^{2}+1}{2 r / a_{1}}+\right. \\
\left.\sqrt{\frac{\left(r / a_{1}\right)^{2}-\left(a_{2} / a_{1}\right)^{2}+1}{2 r / a_{1}}-1}\right] \geqslant 0 \\
\beta=\ln \left[\frac{\left(r / a_{2}\right)^{2}-\left(a_{1} / a_{2}\right)^{2}+1}{2 r / a_{2}}-\right. \\
\left.\sqrt{\frac{\left(r / a_{2}\right)^{2}-\left(a_{1} / a_{2}\right)^{2}+1}{2 r / a_{2}}-1}\right] \leqslant 0
\end{gathered}
$$

其中 $a_{1}, a_{2}$ 分别为两粒子半径, $r$ 为两粒子间的粒心
距离, $a_{0}$ 与 $\alpha, \beta$ 的关系满足式(9):

$$
a_{0}=a_{1} \sinh \alpha=-a_{2} \sinh \beta
$$

对于两个大小形状完全相同的粒子, 其扩散系数的 表达式可以简化为

$$
D(u)=D_{0} \gamma(u)
$$

其中 $u$ 为两粒子的表面最近距离 $h$ 与粒子半径 $a$ 之 比: $u=h / a, \gamma(u)$ 有如下形式:

$$
\begin{aligned}
\gamma(u)= & \frac{4}{3} \sinh (\varphi) \sum_{n=1}^{\infty} \frac{n(n+1)}{(2 n-1)(2 n+3)} \cdot \\
& {\left[\frac{4 \cosh ^{2}\left(n+\frac{1}{2}\right) \varphi+(2 n+1)^{2} \sinh ^{2}(\varphi)}{2 \sinh (2 n+1) \varphi-(2 n+1) \sinh (2 \varphi)}-1\right] }
\end{aligned}
$$

其中 $\varphi=u / 2+1$. Honig 等 ${ }^{[9]}$ 通过简单的拟合, 可以将 上式简化得到 $\gamma(u)$ 的表达式为

$$
\gamma(u)=\frac{6 u^{2}+13 u+2}{6 u^{2}+4 u}
$$

$D(u) / D_{0}$ 的大小随两粒子间距的变化的关系如图 1 所示.

从图 1 中可以看到, 随着两粒子的靠近, 流体动 力学作用的影响随之变大. 在这里需要说明的是, 研 究粒子聚集时, 如果考虑了粒子间的流体动力学作 用, 则必须考虑粒子间存在的吸引势, 否则流体动力 学作用会导致两粒子非常接近时的零扩散,使粒子 无法聚集. 由于考虑的是快聚集, 粒子间不存在排斥 力, 每碰必聚, 而且聚集后粒子不可能分离. 所以我 们在计算中也引人范德华吸引势 $V_{\mathrm{A}}^{[9,29]}$, 其表达式为

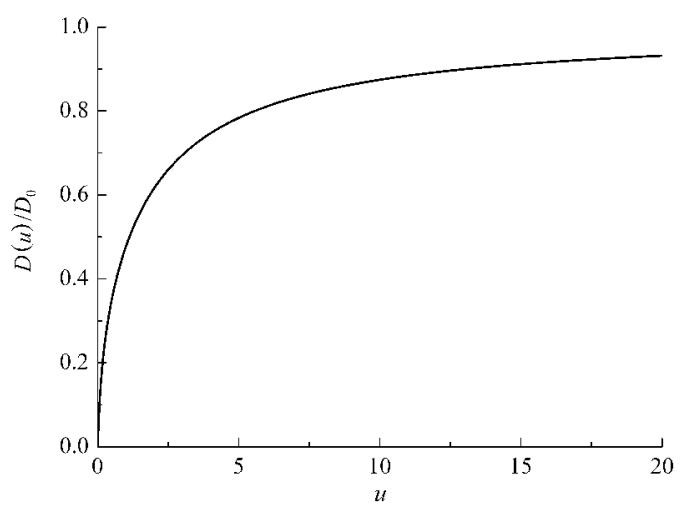

图 1 扩散系数随两粒子之间距离的变化

Fig.1 Diffusion coefficient changes with the distance between two particles

$u=h / a, h$ : the shortest distance between two particles' surfaces, $a$ : radius of particle; $D(u)$ : the diffusion coefficient between two particles with hydrodynamic interaction; $D_{0}$ : the diffusion coefficient of single particle without hydrodynamic interaction 


$$
\begin{aligned}
V_{\mathrm{A}}= & -\frac{H_{\mathrm{a}}}{6}\left[\frac{2 a_{1} a_{2}}{h^{2}+2 a_{1} h+2 a_{2} h}+\frac{2 a_{1} a_{2}}{h^{2}+2 a_{1} h+2 a_{2} h+4 a_{1} a_{2}}+\right. \\
& \left.\ln \left(\frac{h^{2}+2 a_{1} h+2 a_{2} h}{h^{2}+2 a_{1} h+2 a_{2} h+4 a_{1} a_{2}}\right)\right]
\end{aligned}
$$

其中 $H_{\mathrm{a}}$ 为 Hamaker 常数.

通过分析范德华吸引势与粒子距离之间的关系 可知: 粒子间距离较远时, 粒子受到的吸引势的影 响较小, 可以忽略; 粒子间距离较近时, 粒子受范德 华吸引势的影响才较为明显. 因此只考虑粒子间距 离较近的情形, 采用更简化的形式:

$$
V_{\mathrm{A}}=-\frac{H_{\mathrm{a}} a_{1} a_{2}}{6 h\left(a_{1}+a_{2}\right)}
$$

对于直径相等的两个粒子 $\left(a=a_{1}=a_{2}\right)$, 粒子间吸引势 可以表示为

$$
V_{\mathrm{A}}=-\frac{H_{\mathrm{a}}}{12 u}
$$

考虑到在聚集过程中粒子受到重力作用产生沉 降, 根据粒子在某一时刻受到的重力、浮力和阻力达 到平衡, 容易算得半径为 $a$ 的球形粒子沉降速率为

$$
\nu=2|\Delta \rho| g a^{2} / 9 \eta
$$

其中 $\Delta \rho$ 为粒子和溶液的密度差, $g$ 为重力加速度.

有了上面的理论和公式, 在计算机模拟开始之 前还需要给出一些准确的条件和参数. 在初始时刻, 程序中生成大小均一半径为 $a=1.0 \mu \mathrm{m}$ 的球形粒子, 溶液的粘度系数为 $\eta=0.10 \mathrm{~g} \cdot \mathrm{cm}^{-1} \cdot \mathrm{s}^{-1}$, 温度取 $T=300$ $\mathrm{K}$. 此外, 还需引人一些基本假定: 首先, 假设初始存 在的粒子和后来形成的粒子团均为硬球, 即粒子团 之间除接触外没有任何相互作用; 其次, 判断两粒 子团是否聚集的标准是, 对于半径分别为 $a_{i}$ 和 $a_{j}$ 的 粒子团, 当两者的中心距离 $r_{i j}$ 满足 $r_{i j} \leqslant a_{i}+a_{j}$ 时, 即 认为两粒子团已经聚集. 聚集后形成永久性的新粒 子团. 对于新形成粒子团的半径和体积, 采用聚集后 粒子仍融合为球形的假设进行计算: 即一个包含有 $m$ 个粒子的粒子团的半径 $a_{m}$ 可以表示为 $a_{m}=m^{1 / 3} a$; 粒子团的体积 $V_{m}$ 可以表示为 $V_{m}=m V$, 其中 $a$ 和 $V$ 代表单个粒子的半径和体积. 这些条件和假设在以 后的计算中均保持一致.

\section{2 模拟结果与分析}

\section{1 流体动力学作用对粒子聚集的影响}

将式(12)和式(15)用于模拟程序中. 设定聚集开 始溶液中存在 32000 个粒子, 对应粒子的数密度 为 $Z_{0}=1.35 \times 10^{7} \mathrm{~cm}^{-3}$, 体积分数约为 $\phi=5.5 \times 10^{-5}$, 为极 稀溶液. 粒子间吸引势对应的 Hamaker 常数 $H_{\mathrm{a}}$ 为

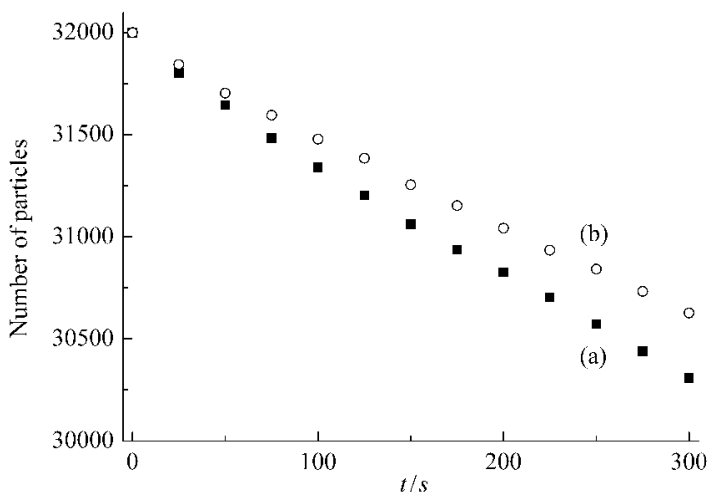

图 2 忽略 $(a)$ 和考虑 $(b)$ 流体动力学作用条件下模拟得到的 粒子数量随时间变化曲线

Fig.2 Time variation of the amount of particles simulated with ignoring (a) and considering (b) hydrodynamic interaction

$10^{-19} \mathrm{~J}$, 分别加人和排除流体动力学作用条件, 得到 聚集开始后 $300 \mathrm{~s}$ 内粒子的聚集曲线(如图 2 所示).

从图 2 可知, 在聚集的初始阶段, 考虑和忽略流 体动力学作用条件下的总粒子数量有很明显的区 别. 考虑流体动力学作用条件下溶液中粒子聚集数 量较少, 剩余数量较多; 忽略流体动力学作用下溶 液中粒子聚集数量较多, 剩余数量较少. 可以看到流 体动力学作用的存在减缓了粒子的聚集.

Honig 等的借鉴了 Overbeek 从流通量推导粒子 聚集速率的方法 ${ }^{[31]}$, 得到了粒子间存在作用势和流 体动力学作用时的聚集速率, 此速率以无量纲参数 $Q$ 表示:

$$
Q=\frac{1}{\int_{0}^{\infty} \gamma(u) \exp \left\{V_{\mathrm{A}}(u) / k_{\mathrm{B}} T\right\} \mathrm{d} u}
$$

不考虑流体动力学作用时, $\gamma(u)=1$; 考虑流体动力学 作用时, $\gamma(u)=\frac{6 u^{2}+13 u+2}{6 u^{2}+4 u}$.

将 Honig ${ }^{[9]}$ 推导的和模拟得到的粒子聚集速率 $Q$ 进 行比较, 结果见表 1.

从表 1 可看到, 计算机模拟中得到的粒子聚集 速率与 Honig 等 ${ }^{[9]}$ 借鉴 $\mathrm{Kruyt}^{[3]}{ }^{[3]}$ 著作中 Overbeek 利

表 1 从 Honig 理论 ${ }^{[9]}$ 推导和计算机模拟得到的 粒子聚集速率 $Q$

Table 1 Particle coagulation rate $Q$ calculated from Honig theory ${ }^{[9]}$ and simulated from our program

\begin{tabular}{llll}
\hline & $Q_{\mathrm{wi}}{ }^{*}$ & $Q_{\mathrm{wo}}{ }^{*}$ & $Q_{\mathrm{wo}}{ }^{* *}$ \\
\hline Honig theory & 3.81 & 5.00 & 2.00 \\
simulation & 3.87 & 4.95 & 2.04 \\
\hline$Q_{\mathrm{wi}}$ : calculated with hydrodynamic interaction, $Q_{\mathrm{wo}}:$ calculated without \\
hydrodynamic interaction, $H_{\mathrm{a}}$ : Hamaker constant; ${ }^{*} H_{\mathrm{a}}=10^{-19} \mathrm{~J},{ }^{*}{ }^{*} H_{\mathrm{a}}=0$
\end{tabular}


用粒子通量推导出的结果基本一致. 说明程序正确, 将两粒子间流体动力学作用的结果用于多粒子聚集 的处理方法也是可行的. 在图 1 中Smoluchowski理 论 (不考虑粒子间作用势和流体动力学作用) 推导 得到的 $Q=2$. 在 Hamaker 常数 $H_{\mathrm{a}}=10^{-19} \mathrm{~J}$ 条件下, 考 虑流体动力学作用时比忽略其作用时 $Q$ 的下降幅 度约为四分之一. 由此可见, 流体动力学作用对粒子 聚集速率的减缓是非常明显的. 尽管此时考虑流体 动力学作用时的 $Q$ 值仍比 Smoluchowski 理论值高, 但是这仅是考虑到粒子间吸引势且吸引势较大时的 结果, 若粒子间吸引势较小或存在排斥势, 则粒子聚 集速率会变得更低. 由此可见, 流体动力学作用可以 明显减缓粒子的聚集, 是导致粒子聚集速率的实验 值低于 Smoluchowski 理论值的一个重要原因.

\section{2 流体动力学作用和重力的塊合影响}

在之前的研究 ${ }^{[20]}$ 和上文中, 我们分别探讨了重 力和流体动力学作用对粒子聚集的影响. 而实际上, 流体动力学作用和重力往往同时存在于粒子聚集的 过程中, 因此在下文中我们将进一步研究两因素耦 合作用下的粒子聚集过程. 显然对这种情况 Honig 等[9]的理论是无法处理的. 在模拟中我们设定初始 时刻溶液中有 64000 个粒子, 对应的粒子数密度为 $Z_{0}=2.7 \times 10^{7} \mathrm{~cm}^{-3}$, 粒子和溶液的密度分别为 $\rho_{\mathrm{s}}=1.1 \mathrm{~g} \cdot$ $\mathrm{cm}^{-3}$ 和 $\rho_{\mathrm{l}}=1.0 \mathrm{~g} \cdot \mathrm{cm}^{-3}$, 密度差为 $\Delta \rho=0.1 \mathrm{~g} \cdot \mathrm{cm}^{-3}$, 粒子 间吸引势对应的 Hamaker 常数仍取 $H_{\mathrm{a}}=10^{-19} \mathrm{~J}$, 模拟 时间为 $10000 \mathrm{~s}$. 由于模拟的是长时间的粒子聚集过 程, 溶液中存在不同大小的粒子聚集的情形, 因此我 们利用式(4)、(14)和(16)分别计算不同大小粒子之 间的流体动力学作用、范德华吸引势和重力沉降的 影响.

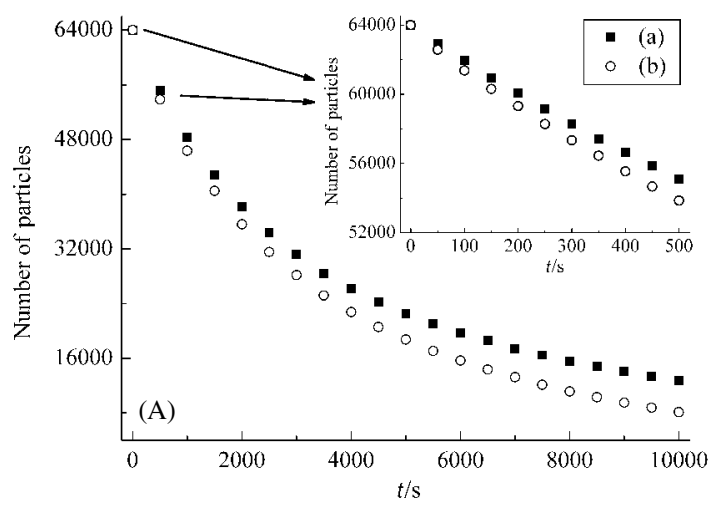

首先, 在两因素耦合作用时流体动力学作用如 何影响粒子聚集是一个十分值得关注的问题, 于是 在 1 倍重力和 5 倍重力条件下, 分别模拟考虑和忽 略流体动力学作用时的粒子聚集过程, 模拟得到的 粒子数量随时间变化的曲线如图 3 所示.

通过图 3(A,B) 可以看到, 无论在 1 倍还是 5 倍 重力条件下, 加人和排除流体动力学作用的两条曲 线从聚集开始阶段就有了很明显的区别: 考虑流体 动力学作用时, 粒子聚集得较慢; 不考虑流体动力 学作用时, 粒子聚集得较快. 此外, 加人和排除流体 动力学作用对应的两曲线的差异, 在聚集的初始阶 段随着时间的增加而增大; 到了聚集后期不再增大 甚至开始减小. 为了解释这种现象, 就必须了解哪些 因素决定了溶液中粒子聚集的快慢. 一方面, 流体动 力学作用的存在会降低两粒子碰撞聚集的可能性. 另一方面, 溶液中粒子的数量也是决定粒子聚集速 率的一个重要因素, 溶液中粒子数量越多, 粒子发生 聚集的可能性也越大. 在粒子聚集的初始时刻,两条 件下溶液中的粒子数量相等. 流体动力学作用存在 时, 由于其阻碍粒子的聚集, 因此粒子数量减少得较 为缓慢; 不存在流体动力学作用时, 粒子数量减少 得较为迅速, 因此两曲线的差异逐渐增大. 经过聚集 一段时间后, 存在流体动力学作用的溶液中剩余粒 子数量较多, 不存在流体动力学作用的溶液中剩余 粒子数量较少. 因此即便考虑到流体动力学作用会 导致粒子聚集变慢, 但由于存在流体动力学作用的 溶液中剩余粒子数量较多的缘故, 粒子聚集得反而 会更快, 于是就出现了两曲线在聚集后期的差异不 再增大甚至减小的情况.

其次, 耦合作用时重力对粒子聚集的影响也是

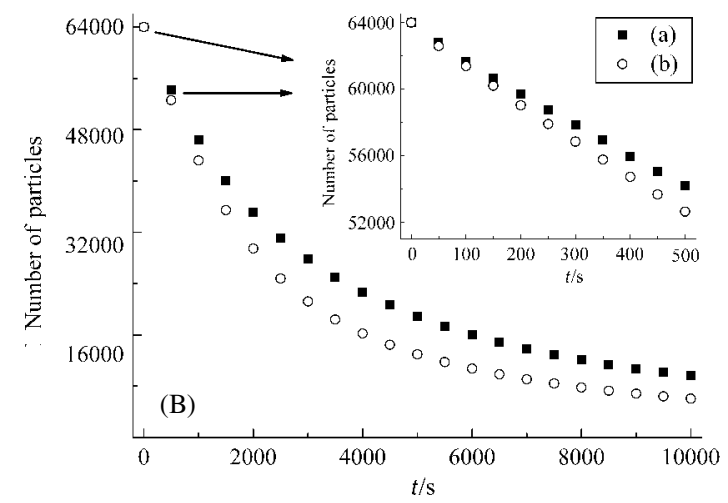

图 31 倍 $(A)$ 和 5 倍 $(B)$ 重力条件下, 考虑 $(a)$ 与忽略 $(b)$ 流体动力学作用时模拟得到的粒子数量随时间变化的曲线

Fig.3 Time variation of the amount of particles simulated with (a) and without (b) hydrodynamic interaction under 1 time (A) and 5 times $(B)$ of gravity condition 

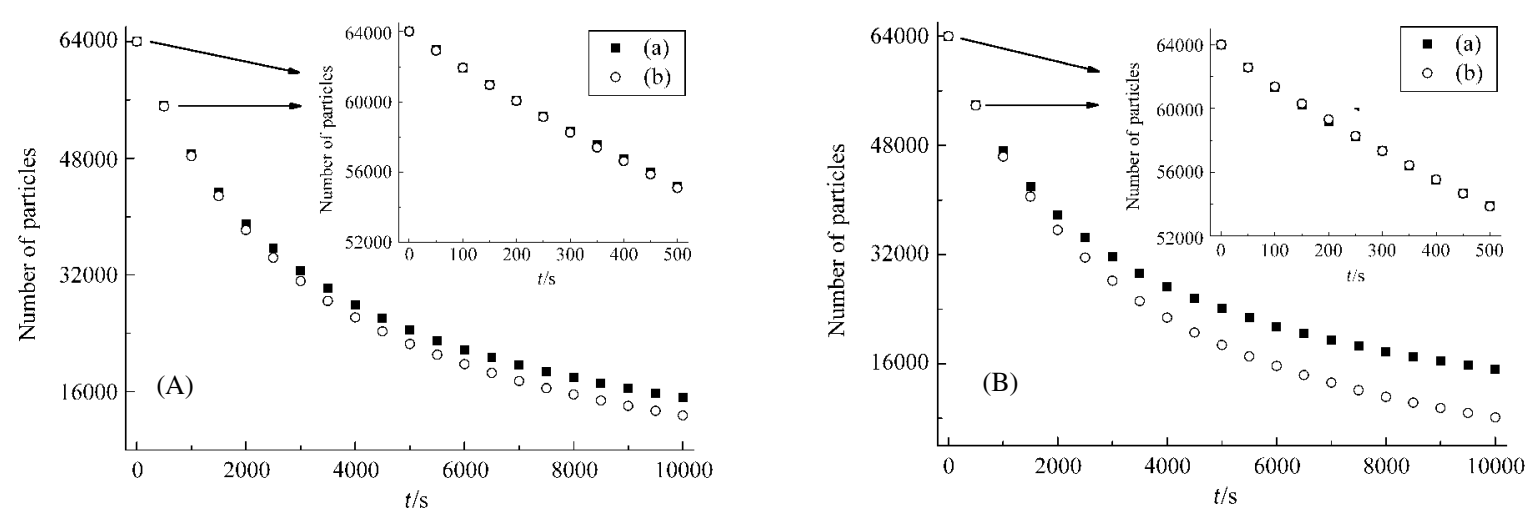

图 4 考虑 $(\mathbf{A})$ 和忽略 $(\mathrm{B})$ 流体动力学作用时, 无重力 $(\mathrm{a})$ 和常重力 $(\mathrm{b})$ 条件下模拟得到的粒子数量随时间变化的曲线

Fig.4 Time variation of the amount of particles simulated under no (a) and normal (b) gravity conditions with (A) and without (B) hydrodynamic interaction

值得关注的问题. 因此, 在考虑和忽略流体动力学作 用两条件下, 分别对无重力和常重力的粒子聚集过 程进行模拟, 得到的粒子数量随时间变化的曲线见 图 4.

在图 4(A, B)中也都可以很明显地观察到, 在聚 集开始的前 $500 \mathrm{~s}$ 内两条粒子数量随时间变化的曲 线几乎没有差异; 之后直至 $10000 \mathrm{~s}$ 时, 两曲线的差 异逐渐增大. 也就是说无论是否考虑粒子间流体动 力学作用, 重力在粒子聚集初期不产生影响, 在聚集 后期加速粒子聚集. 此外, 存在流体动力学作用时, 对应常重力和无重力的两曲线之间出现差异的时间 较晚, 差异较小; 没有流体动力学作用时, 两曲线之 间出现差异的时间较早, 差异较大. 对这种现象, 我 们认为, 不存在流体动力学作用时, 粒子聚集主要有 两种途径: 一方面重力能够增加粒子间相互接近的 频率, 之后通过吸引势的作用无需克服流体动力学 的阻碍就能使粒子之间聚集; 另一方面重力能够直 接导致粒子碰撞而增加粒子聚集的频率, 因此粒子 聚集得较快, 出现大粒子的时间也较早, 由于不同大 小的粒子沉降速度不同, 重力作用显现得较早且更 为明显; 存在流体动力学作用时, 重力并不能直接 导致粒子之间的相互碰撞, 而仅能增加粒子间相互 接近的频率, 还需要依靠粒子间的吸引势克服流体 动力学的阻碍才能聚集, 因此溶液中粒子聚集得较 慢, 出现大粒子的时间较晚, 重力作用显现得也较晚 且更不明显.

\section{3 结 论}

采用布朗动力学模拟方法, 研究了流体动力学
作用对稀释溶液中悬浮粒子聚集过程的影响. 在模 拟计算中, 引人了两粒子之间流体动力学作用对粒 子扩散影响的拟合公式, 模拟结果表明流体动力学 作用的存在减缓了粒子的聚集, 是聚集速率的实验 值小于 Smoluchowski 理论值的一个重要原因. 在粒 子间吸引势不变的前提下, 研究了不同重力条件下 流体动力学作用的影响及忽略和考虑流体动力学作 用条件下重力作用的影响, 得到了两方面的结果. 一 方面, 在不同重力条件下, 考虑流体动力学作用时粒 子聚集得较慢, 忽略流体动力学作用时粒子聚集得 较快. 而考虑和忽略流体动力学作用对应的粒子聚 集数量的差异, 在聚集的初始阶段随着时间的增加 而增大, 而到了后期不再增大甚至开始缩小. 另一方 面, 考虑和忽略流体动力学作用时, 重力均加速粒子 聚集. 考虑流体动力学作用时, 常重力和无重力条件 对应的两曲线之间出现差异的时间较晚, 差异较小; 忽略流体动力学作用时, 两曲线之间出现差异的时 间较早, 差异较大. 对于上述结果, 我们从粒子聚集 动力学的角度出发进行了分析和解释. 这些研究成 果, 可以帮助我们更加明确认识各种因素在聚集过 程中的作用.

\footnotetext{
References

1 von Smoluchowski, M. Physik Z, 1916, 17: 557

2 von Smoluchowski, M. Z. Phys. Chem., 1917, 92: 129

3 Folkersma, R.; Alois, J. G.; van Diemen, A. J.; Stein, H. N. J. Colloid Interface Sci., 1998, 206: 482

4 Folkersma, R.; Stein, H. N. J. Colloid Interface Sci., 1998, 206: 494

5 Folkersma, R.; van Diemen, A. J.; Stein, H. N. Adv. Colloid
} 
Interface Sci., 1999, 83: 71

6 Lamb, H. Hydrodynamics. London: Cambridge University Press, 1975: 1-201

7 Brenner, H. Chem. Engng. Sci., 1961, 16: 242

8 Spielman, L. A. J. Colloid. Interface. Sci., 1970, 33: 562

9 Honig, E. P.; Roebersen, G. J.; Wiersema, P. H. J. Colloid Interface Sci., 1971, 36: 97

10 Crocker, J. C. Phys. Rev. Lett., 1996, 106: 2837

11 German, U. V.; Máximo, G. S.; Jhoan, T. M. Phys. Rev. E, 2003, 68: 061408

12 Zahn, K.; Méndez-Alcaraz, J. M.; Maret. G. Phys. Rev. Lett., 1997, 79: 175

13 Carrasco, B.; de la Torre, J. G.; Zipper, P. Eur. Biophys. J., 1999, 28: 510

14 Carrasco, B.; de la Torre, J. G. Biophys. J., 1999, 75: 3044

15 Carrasco, B.; de la Torre, J. G. J. Chem. Phys., 1999, 111: 4817

16 Allison, S. A.; Chang, E. L.; Schurr, J. M. Chem. Phys., 1979, 38: 29

17 Carter, J. M.; Phillies, G. D. J. J. Phys. Chem., 1985, 89: 5118

18 Felderhof, B. U. Physica A, 1977, 89: 373

19 Felderhof, B. U. J. Phys. A-Math. Gen., 1978, 11: 929
20 Li, X.; Xu, S. H.; Sun, Z. W. Acta Phys. -Chim. Sin., 2009, 25: 207 [李 旭, 徐升华, 孙祉伟. 物理化学学报, 2009, 25: 207]

21 Sun, Z. W.; Chen, Z. Y. Chin. Phys. Lett., 2003, 20: 1634

22 Sun, Z. W.; Liu, J.; Xu, S. H. Chin. Phys. Lett., 2005, 22: 2119

23 Liu, J.; Sun, Z. W.; Yan, A. A. Chin. Phys. Lett., 2005, 22: 3199

24 Sun, Z. W.; Xu, S. H.; Liu, J. J. Jpn. Soc. Microgravity Appl., 2007, 24: 62

25 Pugnaloni, L. A.; Dickinson, E.; Ettelaie, R.; Mackie, A. R.; Wilde, P. J. Adv. Colloid Interface Sci., 2004, 107: 27

26 Adachi, Y. Adv. Colloid Interface Sci., 1995, 56: 1

27 Fukasawa, T.; Adachi, Y. J. Colloid Interface Sci., 2006, 304: 115

28 Allen, M. P.; Tildesley, D. J. Computer simulation of liquids. Oxford: Oxford University Press, 1987: 19-32, 71-108, 147-152, $257-270$

29 Elimelech, M.; Gregory, J.; Jia, X.; Williams, R. A. Particle deposition and aggregation. Woburn, MA: Butterworth-Heinemann Press, 1995: 1-260

30 Rapaport, D. C. The art of molecular dynamics simulation. Cambridge: Cambridge University Press, 1995: 1-435

31 Kruyt, H. R. Colloid science. Amsterdam: Elsevier Publishing Company, 1952(I): 278-286 